\title{
Guaranteeing Multiple Cell Loss Classes in Shared ATM Output Buffer
}

\author{
Hoon Lee \\ Switching Technology Research Laboratories, Korea Telecom \\ 17, Umyon-Dong, Socho-Gu, Seoul, Korea \\ Phone:(82)2-526-6423, Fax:(82)2-526-5419 \\ E-mail:hlee@traffic.kotel.co.kr
}

\begin{abstract}
This paper presents a cell access control scheme for guaranteeing multiple classes of cell loss QoS (Quality of Service) requirements in an output buffer of ATM switch which is shared by multiple connections. First, we propose a class acceptance controller which regulates the acceptance of the cells of QoS classes based on the state of the queue. We consider decision functions for the class acceptance controller with a view to compare their effects to the QoS performance. After that, we present a queueing analysis and derive some important performance measures. Finally, we discuss the implications of the work via numerical experiments.
\end{abstract}

\section{Keywords}

ATM networks, Quality of Service, Priority control

\section{INTRODUCTION}

The B-ISDN (Broadband Integrated Services Digital Networks) technologies based on ATM (Asynchronous Transfer Mode) cell transfer made it possible for various kind of media data to be provided simultaneously in the networks. Typical QoS parameters regarding the ATM sources are the maximum delay, peak-to-peak CDV (Cell Delay Variation) and the cell loss rate (CLR)[3]. The delay and its variation are required to be tightly guaranteed for RT-VBR (Real-time Variable Bit Rate) source, whereas RT-VBR can tolerate a very small value of cell losses.

Since different services in the same RT-VBR require different QoSs, such as the cell loss and delay, the network has to guarantee these different QoSs simultaneously and transparently to the effect of the other services.

The maximum cell delay requirements can be guaranteed by providing a finite queue capacity. On the other hand, the finite system can meet an overflow even if we dimension the queue capacity based on a conservative estimation because the cell input to a buffer is bursty, thus the guarantee of loss rates requires a sophisticated control scheme in the queue before the overflow occurs[8]. In this paper, we mainly consider the cell loss QoS requirements. The cell loss QoS requirement indicates that the mean cell loss of each class due to buffer overflow or any errors must be kept under the target value.

One method to avoid the buffer overflow is to drop cells prior to the occurrence of it by setting one or more thresholds in a buffer and discard a portion of cells

Building QoS into Distributed Systems A. Campbell \& K. Nahrstedt (Eds.)

(c) 1997 IFIP. Published by Chapman \& Hall 
when the queue length exceeds the threshold[5]. In discarding cells, it is favorable for a system to provide a control scheme to drop all cells of one packet or one class rather than to randomly drop cells belonging to different packets or different classes[1]. This corresponds to the class acceptance scheme which we had proposed in the previous work[6].

Turning to the queueing policy, there are two general ways to accomodate the cells of different classes of QoS[2]. One is to provide a separate queue for each class[9], which is usually called a Separate Queue (SpQ) scheme, and the other is to provide a single queue shared by all the classes, which is called the Shared Queue (ShQ) scheme $[5,10]$. Among them, the ShQ scheme can improve the buffer utilization by sharing the queue space, and it is free from the resequencing problem. Thus, the ShQ scheme is more favorable for real-time communication environments. In order to guarantee multiple classes of QoS using ShQ scheme we need a sophisticated cell admission scheme based on the priorities such as the Push-Out (PO) scheme or the Partial buffer Sharing (PbS) scheme[5]. The PO scheme and $\mathrm{PbS}$ scheme are considered and their performance is compared in detail in [7]. Among them, $\mathrm{PbS}$ scheme is considered to be more favorable since it is easy to control and easily implementable. In $\mathrm{PbS}$ scheme, the queue space is divided logically into multiple subspaces and it is partially shared by classes based on the queue occupancy at the cell arrival instant. The basic principle of $\mathrm{PbS}$ scheme is described as follows: When there are QoSs of $K$ classes, one can divide the queue space $B$ (cells) into $K$ subspaces (regions). Whether cells of each class can be accepted or not is decided by the state of the queue at its entering instant : that is, When the queue occupancy is in the range $\left[T_{i}, T_{i+1}\right], i=1, \ldots, K-1, T_{K}=B$, upon arrival of cells, only cells of classes not lower than class $K-i$ is allowed to enter the queue, whereas the other classes are rejected and cells included in those classes are discarded in that time slot.

In this paper, we consider the same $\mathrm{PbS}$ scheme. Upon determining the decision function, we propose a new method: first we set a base threshold, next we determine calibrating thresholds (the discussion in detail will be given in section 3). We assumed five cell loss classes, which is based on the linguistic representation of the degree of stringency of the cell loss requirement: very stringent, stringent, moderate, loose, and very loose.

\section{SYSTEM MODEL}

Let us consider an output multiplexer of ATM switch which accommodates $K$ different kinds of source groups with corresponding $K$ heterogeneous cell loss classes.

We call a source which has cell loss class $k, k \in\{1,2, \ldots, K\}$, as class $k$ source according to the stringency of the cell loss requirement. The number of class $k$ sources is assumed to be $N_{k}$. We assume that the smaller the class number the higher the priority. That is, if $K=5$, the class $1,2,3,4$, and 5 corresponds to the very stringent, stringent, moderate, loose, and very loose class, respectively.

We put a Class Acceptance (CA) controller in front of the queue. The queue is finite with capacity $B$ (cells), and the cell input and output operations are performed based on a time slot. A time slot is a time unit to serve a fixed length cell, and the time slots of different cell loss classes are synchronized one another.

The queue state is observed at the beginning of every time slot, and it is transferred to the CA controller. The CA controller determines the number of classes 
that can be allowed to input the cells into the queue based on the decision function. The decision function is called the Class Acceptance (CA) function, and it is denoted by $y(x)$.

The CA controller accepts cells from the sources whose classes are not greater than class $y(n)$ when the queue occupancy at the beginning of that time slot is observed to be $n$, whereas the cells generated from the sources of classes greater than $y(n)$ are discarded in that time slot.

\section{CLASS ACCEPTANCE FUNCTION}

We propose that $y(x)$ can be determined by considering the following three conditions;

- Extreme conditions : The CA function satisfies the extreme conditions $y(0)=K$ and $y(B)=1$. The condition $y(0)=K$ indicates that, when the queue length at the beginning of the time slot is 0 , the CA controller accepts cells from all the classes. The condition $y(B)=1$ indicates that, when the queue length is $B$, cells from only the highest class, viz., cells from the class 1 , can enter the queue. Note that $y(B)=1$ holds because at least a cell can enter the queue if we assume the departure first rule in a queue.

- Safeguard regions : The CA controller has to operate, to a degree, in favor of higher QoS classes, but it should also guarantee the best-effort QoSs to the lower QoS classes. To this end, the CA function should be generous to the lower classes when the queue occupancy is not too high, whereas it should be gradually and smoothly become stringent to the lower classes as the queue builds up. When the queue length approaches $B$, the control has to be very strict even to the higher classes.

In order to determine an optimal CA function which satisfies the required cell loss rate for each class, we investigate some heuristic decision functions. First, let us consider a uniform partition of a queue space. A typical curve for uniform partition is illustrated in Fig.1 for $K=5$. In Fig.1, we also consider another partition, that is, a partition with safeguard. A quantitative comparison of two cases will be given

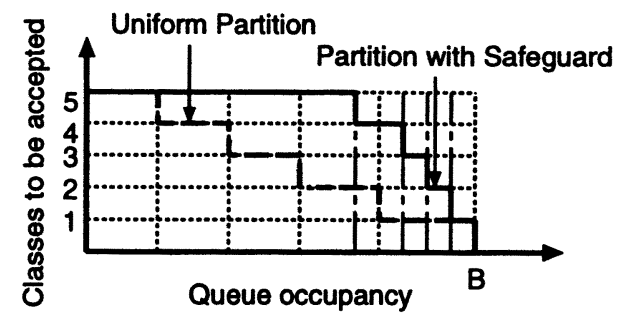

Fig.1. Partition of a queue.

in section 5 .

Let us put our objective to guaranteeing the cell loss requirement of the most stringent class. Let us determine the threshold for most stringent cell loss class based on the cell loss requirement of that class, which we call a base threshold, by 
using the result of binary threshold for two-class system[8]. After that, we determine the remaining thresholds in a heuristic manner. This scheme is reasonable because we have to guarantee the most stringent class in the highest priority.

First, let us determine the base threshold. It is known that the limiting queueing behavior of an ATM switch located arbitrarily in the middle of the network is accurately modeled by an $\mathrm{M} / \mathrm{D} / 1$ type queueing model[8].

For the system with sources described by the mean arrival rate and CLR required by it we can obtain the queue capacity which guarantees the CLR under the given cell arrival rate by using the result of $M / D / 1$ type queueing model[8]. The queue capacity in this case corresponds to the base threshold in our discussion. That is, if we are given that the mean arrival rate of the most stringent class is $\lambda_{1}$ and the CLR of which is given by $\phi_{1}$, then we can obtain the base threshold $T_{b}$ given by

$$
T_{b}=\frac{\ln \phi_{1}-\ln (1-\lambda)\left(e^{\lambda_{1}}-1-2 \lambda_{1} / 3\right)}{\ln \left(e^{\lambda}-1-2 \lambda / 3\right)-\ln \left(e^{\lambda_{1}}-1-2 \lambda_{1} / 3\right)}
$$

where $\lambda$ is the total cell arrival rate including the most stringent class. The remaining thresholds are determined by two heuristics described above.

\section{ANALYSIS AND QOS PERFORMANCE}

\subsection{Queue analysis}

First, let us define the arrival process and the input process as follows. The arrival process is an aggregated source process which is generated from the superposition of $K$ source classes, whereas the input process is a process which is composed of cells that pass through the CA controller. Thus, if we use the term "arrival ", it is related to the input side of CA controller, whereas the term "input " is related to the output side of the CA controller, which is the input to a queue.

Let us assume that cell arrivals from sources of the same QoS class are independent and identically distributed (i.i.d.) with the cell arrival probability $\sigma_{k}, k \in\{1,2, \ldots$, $K\}$. Let $a_{k}$ be the number of cells generated from the class $k$ source in an arbitrary time slot, and let $a_{\triangleleft y(x)}$ be the total number of cells that are allowed to enter the queue given that the queue occupancy at the beginning of that time slot is $x$. Note that the total number of cells that can enter a queue in an arbitrary time slot depends on the queue state at the beginning of that time slot. That is, if the CA controller find the queue length to be $x$, then the sources of only upper $y(x)$ classes (i.e., sources of class $1,2, \ldots, y(x)$ ) is allowed to enter the queue in that time slot. Thus, $a_{\triangleleft y(x)}$ is given as follows.

$$
a_{\triangleleft y(x)}=\sum_{k=1}^{y(x)} a_{k} .
$$

If we let

$$
\alpha_{\triangleleft y(x)}(m)=\operatorname{Pr}\left\{a_{\triangleleft y(x)}=m\right\}, m=0,1, \ldots, \sum_{l=1}^{y(x)} N_{l}
$$


be the probability of $m$ cell inputs from an aggregate of sources of upper $y(x)$ classes in a time slot, it is the probability that the total number of cells generated by the accepted sources is $m$ given that the queue state is $x$ at the beginning of that time slot. Let $\alpha_{k}(m)$ be the probability that the number of cell arrivals from the source class $k$ is $m, 0 \leq m \leq N_{k}$. Then, we obtain

$$
\alpha_{\triangleleft y(x)}(m)=\alpha_{1} \star \alpha_{2} \star \ldots \star \alpha_{y(x)}(m),
$$

where $\star$ denotes the convolution. The mean arrival rate of superposed arrival process is defined by $\lambda_{\triangleleft K}=\sum_{k=1}^{K} \lambda_{k}$, where $\lambda_{k}$ is the mean arrival rate of the class $k$, since we assumed the independency between different classes. The system stability condition states that $\lambda_{\triangleleft K}<1$.

Let us consider an arbitrary time slot $i$, and note that a batch of cell inputs to a queue is fed from the higher $y\left(x_{i}\right)$ classes given that the queue state at the beginning of that time slot is $x_{i}$. A cell departs from the queue immediately after the beginning of a time slot if there is any cell in that queue.

Let $a_{\triangleleft y\left(x_{i}\right)}$ denotes the number of cells that enter the queue before the end of time slot $i$. The service rule is FIFO (First-In-First-Out) and the service order for the simultaneously entered cells is random. Thus, the input-output order seen from the queue is departure first. Then, we can obtain a formula for the queue length between the two consecutive time slots $i$ and $i+1$ as follows :

$$
x_{i+1}=\left[\left(x_{i}-1,0\right)^{+}+a_{\triangleleft y\left(x_{i}\right)}, B\right]^{-},
$$

where $[\tau, \omega]^{-}$and $[\tau, \omega]^{+}$indicates the minimum and maximum of $\tau$ and $\omega$, respectively.

From the above discussion, we know that the sequence $\mathbf{x}=\left\{x_{i} ; i \geq 1\right\}$ constitutes a homogeneous Markov chain[4] with the state transition probability defined by

$$
p(m, n)=\operatorname{Pr}\left\{x_{i+1}=n \mid x_{i}=m\right\}, 0 \leq m, n \leq B .
$$

Since the source classes that are allowed to enter the queue is determined by the state of the queue at the beginning of that time slot, we can write the formula (4) as follows :

$$
p(m, n)=\operatorname{Pr}\left\{\left[\left(x_{i}-1,0\right)^{+}+a_{\triangleleft y\left(x_{i}\right)}, B\right]^{-}=n \mid x_{i}=m\right\},
$$

which is rewritten by

$$
p(m, n)=\operatorname{Pr}\left\{\left[(m-1,0)^{+}+a_{\triangleleft y(m)}, B\right]^{-}=n\right\} .
$$

From (3), the formula (5) can be written as follows :

$$
p(m, n)= \begin{cases}\alpha_{\triangleleft y(0)}(n), & m=0 \& 0 \leq n \leq B-1, \\ \alpha_{\triangleleft y(0)}(B \triangleright), & m=0 \& n=B, \\ \alpha_{\triangleleft y(m)}(n-m+1), & m>0 \& 0 \leq n \leq B-1, \\ \alpha_{\triangleleft y(m)}((B-m+1) \triangleright), & m>0 \& n=B,\end{cases}
$$

where the notation $Z \triangleright$ indicates the number greater than or equal to $Z$.

In order to represent the state transition probability in more explicit formula, let us recall that we assumed five cell loss classes; from class 1 to class 5 , and correspondingly four thresholds, $T_{1}, T_{2}, T_{3}$, and $T_{4}$, can be assumed. 
First, we rewrite the state transition probability as follows : For $m=0$, we have

$$
p(0, n)= \begin{cases}p_{n}, & 0 \leq n \leq B-1, \\ P_{B}, & n=B\end{cases}
$$

where $p_{n}=\operatorname{Pr}\left\{a_{\triangleleft y(0)}=n\right\}=\alpha_{\triangleleft 5}(n)$ and $P_{B}=\operatorname{Pr}\left\{a_{\triangleleft 5} \geq B\right\}=1-\sum_{n=0}^{B-1} p_{n}$.

For $1 \leq m \leq B$, we have

$$
p(m, n)= \begin{cases}\tilde{p}_{n-m+1}, & m-1 \leq n \leq B-1, \\ \tilde{P}_{B-m+1}, & n=B \\ 0, & \text { otherwise }\end{cases}
$$

where $\tilde{p}_{n-m+1}=\operatorname{Pr}\left\{a_{\triangleleft y(m)}=n-m+1\right\}=\alpha_{\triangleleft y(m)}(n-m+1)$, and it is redefined as follows :

$$
\tilde{p}_{n-m+1}= \begin{cases}p_{n-m+1}, & 1 \leq m \leq T_{1}, \\ q_{n-m+1}, & T_{1}+1 \leq m \leq T_{2}, \\ r_{n-m+1}, & T_{2}+1 \leq m \leq T_{3}, \\ s_{n-m+1}, & T_{3}+1 \leq m \leq T_{4}, \\ t_{n-m+1}, & T_{4}+1 \leq m \leq B,\end{cases}
$$

where $p_{l}=\operatorname{Pr}\left\{a_{\triangleleft 5}=l\right\}=\alpha_{\triangleleft 5}(l), q_{l}=\operatorname{Pr}\left\{a_{\triangleleft 4}=l\right\}=\alpha_{\triangleleft 4}(l), r_{l}=\operatorname{Pr}\left\{a_{\triangleleft 3}=l\right\}=\alpha_{\triangleleft 3}(l)$, $s_{l}=\operatorname{Pr}\left\{a_{\triangleleft 2}=l\right\}=\alpha_{\triangleleft 2}(l)$, and $t_{l}=\operatorname{Pr}\left\{a_{\triangleleft 1}=l\right\}=\alpha_{\triangleleft 1}(l)$.

On the other hand, $\tilde{P}_{B-m+1}=\operatorname{Pr}\left\{a_{\triangleleft y(m)} \geq B-m+1\right\}$, where $\tilde{P}_{B-m+1}=1-\sum_{n=0}^{B-m} \tilde{p}_{n}$, which can be rewritten in the same way:

$$
\tilde{P}_{B-m+1}= \begin{cases}P_{B-m+1}, & 1 \leq m \leq T_{1}, \\ Q_{B-m+1}, & T_{1}+1 \leq m \leq T_{2}, \\ R_{B-m+1}, & T_{2}+1 \leq m \leq T_{3}, \\ S_{B-m+1}, & T_{3}+1 \leq m \leq T_{4}, \\ T_{B-m+1}, & T_{4}+1 \leq m \leq B,\end{cases}
$$

where $P_{l}=\operatorname{Pr}\left\{a_{\triangleleft 5} \geq l\right\}, Q_{l}=\operatorname{Pr}\left\{a_{\triangleleft 4} \geq l\right\}, R_{l}=\operatorname{Pr}\left\{a_{\triangleleft 3} \geq l\right\}, S_{l}=\operatorname{Pr}\left\{a_{\triangleleft 2} \geq l\right\}$, and $T_{l}=\operatorname{Pr}\left\{a_{\triangleleft 1} \geq l\right\}$.

Then, we can obtain the state transition matrix of the queue, $\mathbf{M}=(p(m, n))$, $0 \leq m, n \leq B$, and if we let $\pi_{x}$ be the probability that the queue length equals $x$ in equilibrium and denote the stationary probability vector of the Markov chain by $\pi=\left(\pi_{0}, \pi_{1}, \ldots, \pi_{B}\right)$, then $\pi$ is the unique solution of the balance equation given by

$$
\pi \mathrm{M}=\pi, \pi e=1,
$$

where the latter equation is the normalization condition and $e$ is the $(B+1) \times 1$ column vector with all elements equal to one.

\subsection{Performance measures}

Rejection probability for class $k$, where $k>1$, source is defined as a steady state probability that the class $k$ source finds the queue occupancy in the range $\left[T_{K+1-k}+\right.$ 
$1, B], k>1$. Note that class 1 source is free from rejection. Thus, we obtain the rejection probability of class $k$ source, $P r_{r e j}^{k}, k>1$, as follows.

$$
\operatorname{Pr}_{r e j}^{k}=\sum_{x=T_{K+1-k}+1}^{B} \pi_{x}, \quad k>1
$$

On the other hand, the probability of no rejection, $P r_{n o r e j}$, i.e., the probability that all the classes can be accepted, is given by

$$
P r_{n o r e j}=\sum_{x=0}^{T_{1}} \pi_{x}
$$

The cell loss in a queue results from two different reasons : The cell loss due to class rejection by CA controller and the cell loss due to buffer overflow after the cell has been allowed to enter the queue. The former corresponds to the rate of rejected (discarded) cells, whereas the latter corresponds to that of the overflowed ones. We consider these two cases in the following.

Let $L r_{r e j}^{k}$ be the cell loss rate of class $k, k>1$, source due to class rejection, and call it a rejection loss rate. Then, we obtain a formula for the rejection loss rate for class $k, k>1$, as follows.

$$
L r_{r e j}^{k}=\frac{1}{\lambda_{k}} \sum_{x=T_{K+1-k+1}}^{B} \pi_{x} \sum_{l_{k}(x)=1}^{N_{k}} l_{k}(x) \mathcal{A}_{l_{k}}(x), \quad k>1,
$$

where $\mathcal{A}_{l_{k}}(x)$ is the probability that $l_{k}(x)$ cells of class $k$ source arrive in a time slot given that the queue occupancy was $x$ at the beginning of that time slot.

Let $L r_{\text {ovf }}^{k}$ be the cell loss rate of class $k, k>0$, source due to buffer overflow, and call it an overflow loss rate. The cell loss of class $k$ source due to buffer overflow depends on the total number of the aggregated inputs as well as the number of the vacant space in the queue at its input instant. If the queue length in that instant is $x, x>0$, and the total number of the aggregated cell inputs is $l(x)$, and if $l(x)>B-x+1$, then $l(x)-B+x-1$ cells are lost due to buffer overflow. When $x=0$ in that instant, the total number of the aggregated cell inputs is $l(0)$, and if $l(0)>B$, then $l(0)-B$ cells are lost due to buffer overflow. We let $o v(x)=l(x)-B+x-1, x>0$ and $o v(0)=l(0)-B$ in the sequel. We assume that among $l(x)$ cells the class $k$ cells are $l_{k}(x)$. Since the sources of each class are independent, the probability of cell loss for the class $k$ source is $\frac{l_{k}(x)}{l(x)}$ that of the total cell loss probability.

Since the aggregated cell arrivals from one class are independent of the aggregated cell arrivals from the remaining classes, the probability that $l_{k}(x)$ cells arrive from a set of class $k$ sources is given by $\operatorname{Pr}\left\{a_{k}=l_{k}(x) \mid a_{\text {tot }}=l(x)\right\}=\operatorname{Pr}\left\{a_{k} \|_{k}(x)\right\}=$ $\mathcal{A}_{l_{k}}(x)$, where $a_{k}$ is the number of cells arrived by source class $k$ and $a_{t o t}$ stands for the number of cell arrivals from total accepted sources.

Then, we obtain the formula for the cell overflow loss rate for a cell of class $k$, $k \in\{1,2,3,4,5\}$, source as follows. For class 1 cell, we obtain

$$
L r_{o v f}^{1}=\frac{1}{\lambda_{\triangleleft 5}} \pi(0) \sum_{l(0)=B+1}^{N_{\triangleleft 5}}\left\{o v(0) \mathcal{A}_{l}(0) \sum_{l_{1}(0)=1}^{N_{1}} \frac{l_{1}(0)}{l(0)} \mathcal{A}_{l_{1}}(0)\right\}
$$




$$
\begin{aligned}
& +\frac{1}{\lambda_{\triangleleft 5}} \sum_{x=1}^{T_{1}} \pi(x) \sum_{l(x)=B-x+2}^{N_{\triangleleft 5}}\left\{o v(x) \mathcal{A}_{l}(x) \sum_{l_{1}(x)=1}^{N_{1}} \frac{l_{1}(x)}{l(x)} \mathcal{A}_{l_{1}}(x)\right\} \\
& +\frac{1}{\lambda_{\triangleleft 4}} \sum_{x=T_{1}+1}^{T_{2}} \pi(x) \sum_{l(x)=B-x+2}^{N_{\triangleleft 4}}\left\{o v(x) \mathcal{A}_{l}(x) \sum_{l_{1}(x)=1}^{N_{1}} \frac{l_{1}(x)}{l(x)} \mathcal{A}_{l_{1}}(x)\right\} \\
& +\frac{1}{\lambda_{\triangleleft 3}} \sum_{x=T_{2}+1}^{T_{3}} \pi(x) \sum_{l(x)=B-x+2}^{N_{\triangleleft 3}}\left\{o v(x) \mathcal{A}_{l}(x) \sum_{l_{1}(x)=1}^{N_{1}} \frac{l_{1}(x)}{l(x)} \mathcal{A}_{l_{1}}(x)\right\} \\
& +\frac{1}{\lambda_{\triangleleft}} \sum_{x=T_{3}+1}^{T_{4}} \pi(x) \sum_{l(x)=B-x+2}^{N_{\triangleleft 2}}\left\{o v(x) \mathcal{A}_{l}(x) \sum_{l_{1}(x)=1}^{N_{1}} \frac{l_{1}(x)}{l(x)} \mathcal{A}_{l_{1}}(x)\right\} \\
& +\frac{1}{\lambda_{\triangleleft 1}} \sum_{x=T_{4}+1}^{B} \pi(x) \sum_{l(x)=B-x+2}^{N_{\triangleleft 1}}\left\{o v(x) \mathcal{A}_{l}(x) \sum_{l_{1}(x)=1}^{N_{1}} \frac{l_{1}(x)}{l(x)} \mathcal{A}_{l_{1}}(x)\right\},
\end{aligned}
$$

where $l(0) \geq l_{1}(0), l(x) \geq l_{1}(x), \lambda_{\triangleleft k}=\sum_{i=1}^{k} \lambda_{i}, N_{\triangleleft k}=\sum_{i=1}^{k} N_{i}$ and $\mathcal{A}_{l}(x)$ is the probability that a total of $l(x)$ cells enter in a slot given that the queue occupancy was $x$ at the beginning of that slot. Note in the above that $N_{\triangleleft 1}$ is identical to $N_{1}$. In the same manner, $\lambda_{\triangleleft 1}$ is equivalent to $\lambda_{1}$.

For class 2 cell, we obtain

$$
\begin{aligned}
& L r_{o v f}^{2}=\frac{1}{\lambda_{\triangleleft 5}} \pi(0) \sum_{l(0)=B+1}^{N_{\triangleleft 5}}\left\{o v(0) \mathcal{A}_{l}(0) \sum_{l_{2}(0)=1}^{N_{2}} \frac{l_{2}(0)}{l(0)} \mathcal{A}_{l_{2}}(0)\right\} \\
& +\frac{1}{\lambda_{\triangleleft 5}} \sum_{x=1}^{T_{1}} \pi(x) \sum_{l(x)=B-x+2}^{N_{\triangleleft 5}}\left\{o v(x) \mathcal{A}_{l}(x) \sum_{l_{2}(x)=1}^{N_{2}} \frac{l_{2}(x)}{l(x)} \mathcal{A}_{l_{2}}(x)\right\} \\
& +\frac{1}{\lambda_{\triangleleft 4}} \sum_{x=T_{1}+1}^{T_{2}} \pi(x) \sum_{l(x)=B-x+2}^{N_{\triangleleft 4}}\left\{o v(x) \mathcal{A}_{l}(x) \sum_{l_{2}(x)=1}^{N_{2}} \frac{l_{2}(x)}{l(x)} \mathcal{A}_{l_{2}}(x)\right\} \\
& +\frac{1}{\lambda_{\triangleleft 3}} \sum_{x=T_{2}+1}^{T_{3}} \pi(x) \sum_{l(x)=B-x+2}^{N_{\triangleleft 3}}\left\{o v(x) \mathcal{A}_{l}(x) \sum_{l_{2}(x)=1}^{N_{2}} \frac{l_{2}(x)}{l(x)} \mathcal{A}_{l_{2}}(x)\right\} \\
& +\frac{1}{\lambda_{\triangleleft 2}} \sum_{x=T_{3}+1}^{T_{4}} \pi(x) \sum_{l(x)=B-x+2}^{N_{\triangleleft 2}}\left\{o v(x) \mathcal{A}_{l}(x) \sum_{l_{2}(x)=1}^{N_{2}} \frac{l_{2}(x)}{l(x)} \mathcal{A}_{l_{2}}(x)\right\},
\end{aligned}
$$

where $l(0) \geq l_{2}(0)$ and $l(x) \geq l_{2}(x)$.

For class 3 cell, we obtain

$$
\begin{aligned}
& L r_{o v f}^{3}=\frac{1}{\lambda_{\triangleleft 5}} \pi(0) \sum_{l(0)=B+1}^{N_{\triangleleft 5}}\left\{o v(0) \mathcal{A}_{l}(0) \sum_{l_{3}(0)=1}^{N_{3}} \frac{l_{3}(0)}{l(0)} \mathcal{A}_{l_{3}}(0)\right\} \\
& +\frac{1}{\lambda_{\triangleleft 5}} \sum_{x=1}^{T_{1}} \pi(x) \sum_{l(x)=B-x+2}^{N_{\triangleleft 5}}\left\{o v(x) \mathcal{A}_{l}(x) \sum_{l_{3}(x)=1}^{N_{3}} \frac{l_{3}(x)}{l(x)} \mathcal{A}_{l_{3}}(x)\right\}
\end{aligned}
$$




$$
\begin{aligned}
& +\frac{1}{\lambda_{\triangleleft 4}} \sum_{x=T_{1}+1}^{T_{2}} \pi(x) \sum_{l(x)=B-x+2}^{N_{\triangleleft 4}}\left\{o v(x) \mathcal{A}_{l}(x) \sum_{l_{3}(x)=1}^{N_{3}} \frac{l_{3}(x)}{l(x)} \mathcal{A}_{l_{3}}(x)\right\} \\
& +\frac{1}{\lambda_{\triangleleft 3}} \sum_{x=T_{2}+1}^{T_{3}} \pi(x) \sum_{l(x)=B-x+2}^{N_{\triangleleft 3}}\left\{o v(x) \mathcal{A}_{l}(x) \sum_{l_{3}(x)=1}^{N_{3}} \frac{l_{3}(x)}{l(x)} \mathcal{A}_{l_{3}}(x)\right\}
\end{aligned}
$$

where $l(0) \geq l_{3}(0)$ and $l(x) \geq l_{3}(x)$.

For class 4 cell, we obtain

$$
\begin{aligned}
& L r_{o v f}^{4}=\frac{1}{\lambda_{\triangleleft 5}} \pi(0) \sum_{l(0)=B+1}^{N_{\triangleleft 5}}\left\{o v(0) \mathcal{A}_{l}(0) \sum_{l_{4}(0)=1}^{N_{4}} \frac{l_{4}(0)}{l(0)} \mathcal{A}_{l_{4}}(0)\right\} \\
& +\frac{1}{\lambda_{\triangleleft 5}} \sum_{x=1}^{T_{1}} \pi(x) \sum_{l(x)=B-x+2}^{N_{\triangleleft 5}}\left\{o v(x) \mathcal{A}_{l}(x) \sum_{l_{4}(x)=1}^{N_{4}} \frac{l_{4}(x)}{l(x)} \mathcal{A}_{l_{4}}(x)\right\} \\
& +\frac{1}{\lambda_{\triangleleft 4}} \sum_{x=T_{1+1}}^{T_{2}} \pi(x) \sum_{l(x)=B-x+2}^{N_{\triangleleft 4}}\left\{o v(x) \mathcal{A}_{l}(x) \sum_{l_{4}(x)=1}^{N_{4}} \frac{l_{4}(x)}{l(x)} \mathcal{A}_{l_{4}}(x)\right\}
\end{aligned}
$$

where $l(0) \geq l_{4}(0)$ and $l(x) \geq l_{4}(x)$.

For class 5 cell, we obtain

$$
\begin{aligned}
& L r_{o v f}^{5}=\frac{1}{\lambda_{\triangleleft 5}} \pi(0) \sum_{l(0)=B+1}^{N_{\triangleleft 5}}\left\{o v(0) \mathcal{A}_{l}(0) \sum_{l_{5}(0)=1}^{N_{5}} \frac{l_{5}(0)}{l(0)} \mathcal{A}_{l_{5}}(0)\right\} \\
& +\frac{1}{\lambda_{\triangleleft 5}} \sum_{x=1}^{T_{1}} \pi(x) \sum_{l(x)=B-x+2}^{N_{\triangleleft 5}}\left\{o v(x) \mathcal{A}_{l}(x) \sum_{l_{5}(x)=1}^{N_{5}} \frac{l_{5}(x)}{l(x)} \mathcal{A}_{l_{5}}(x)\right\}
\end{aligned}
$$

where $l(0) \geq l_{5}(0)$ and $l(x) \geq l_{5}(x)$.

\section{NUMERICAL RESULTS}

Let us assume that the cell generating process follows a general independent burst process, and let it a binomial process $b\left(N_{k}, \sigma_{k}\right), k \in\{1,2, \ldots, K\}$. For class $k$ source, the probability of $m_{k}$ cell arrivals per time slot by class $k$ sources is $\alpha_{k}\left(m_{k}\right)=$ $\left(\begin{array}{l}N_{k} \\ m_{k}\end{array}\right) \sigma_{k}^{m_{k}} \zeta_{k}^{N_{k}-m_{k}}, \zeta_{k}=1-\sigma_{k}$, and the mean arrival rate is $\lambda_{k}=N_{k} \sigma_{k}$. Let $K=5$ as we assumed before, and the number of sources for each class is the same and $N_{k}=10$ for class $k=1,2,3,4$ and 5 .

We assume that the queue capacity is small and it is given by $B=20$. First, let us determine the base threshold. If we assume that the cell arrival rate of the most stringent class is $\lambda_{1}=0.1$ and the required CLR for that class is assumed to be $10^{-12}$, and if we use the formula (1), the base threshold is obtained to be $T_{b}=14$, which corresponds to $T_{1}$.

Next, the decision function is shown in Fig.2, where the base threshold is 14 and the other thresholds are given by appropriate proportions. These assumptions are effective throughout this section. Fig. 3 illustrates the mean class rejection rate for classes 2, 3, 4 and 5 as a function of the $\sigma_{2}$, the cell arrival probability of class 2 


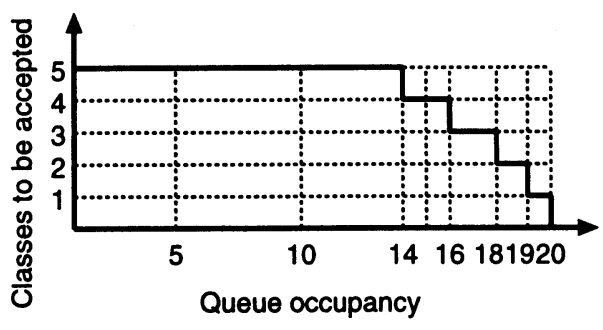

Fig.2. Partition with base threshold of 14 .

source. The relationships between $\sigma_{i}, i=1,2,3,4,5$ are given as follows: $\sigma_{1}=\sigma_{5}=$ $0.010, \sigma_{2}=\sigma_{4}=[0.005,0.011]$ with an increment of unit 0.001 , and $\sigma_{3}=\sigma_{2} * 1.5$. This assumption holds throughout this section. Via preliminary computation we knew that the CLR of $10^{-12}$ for class 1 source can not be guaranteed if the mean arrival rate of the class 2 source exceeds 0.011 .

In Fig. 3 and its subsequent figures, the acronym UP and PS indicates the Uniform Partition (UP) and Partition with Safeguard (PS) as we had defined in section 3. Note that class 1 source is not rejected. We also find that the UP scheme ex-

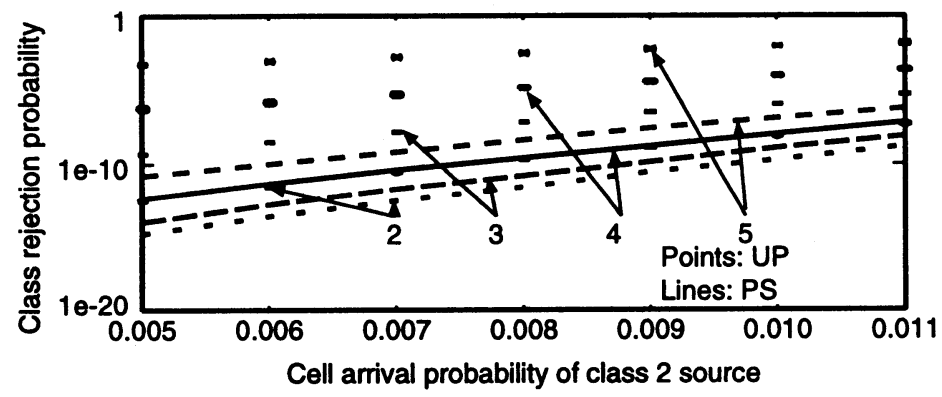

Fig.3. Mean class rejection rate.

tremely discards the cells with low classes compared with the PS scheme. Thus, we can conclude that the UP scheme over-regulates the cell ingress to a queue.

Fig.4 illustrates the mean rejection loss rates for classes 2,3,4 and 5 . The rejection loss results from the class rejection, which follows the same trend as that of Fig.3. This is easily expected. Fig.5 illustrates the mean overflow loss rate for classes 2,3,4 and 5. As we can find from Fig.5, the overflow of cells under the UP scheme is much less than that by PS scheme, which is self clear in that the UP scheme over regulates the cell ingression prior to the occurrence of the overflow compared with the PS scheme. We can find the overall cell loss rate from the last two figures. 


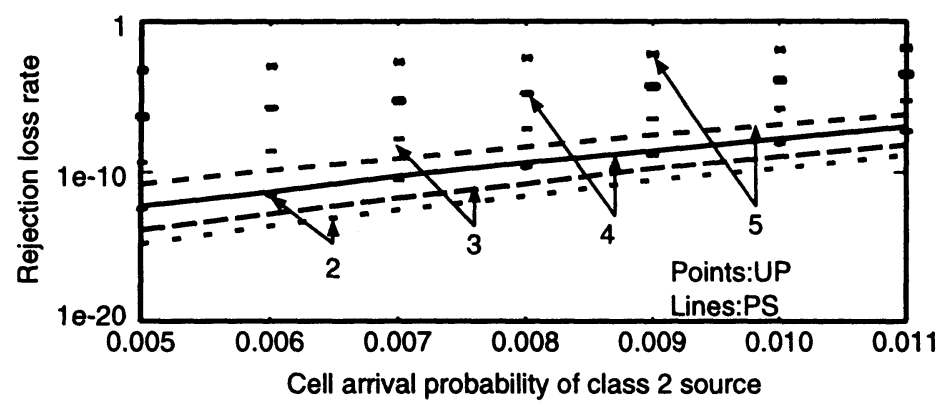

Fig.4. Mean rejection loss rate.

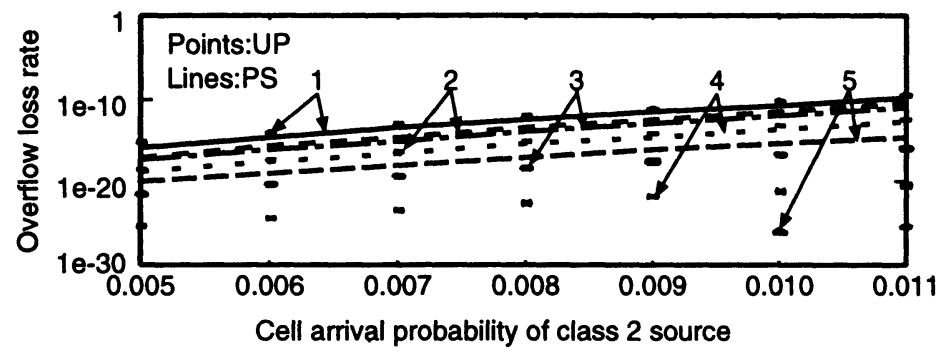

Fig.5. Mean overflow rate.

\section{CONCLUSION}

The main contributions of this paper are as follows : First, we proposed a cell access control scheme for guaranteeing multiple cell loss QoS in shared ATM output buffer by analytical model. Second, as an extension to the previous paper[6], we introduced the concept of the base threshold in a queue which plays a role of guaranteeing most stringent cell loss requirement exclusively from the remaining classes. This base threshold also acts as a marginal limit for guaranteeing no cell discard to all the classes, which would have an important implication. Finally, by numerical experiments we illustrated the effect of cell acceptance control in a queue to the QoS performance, from which we can estimate the maximum allowable cell input rate of associated QoS classes which guarantees the most stringent cell loss requirements in the system. Our future research area includes the modification of the CA function as well as the extension of the source model to a correlated one.

\section{References}

[1] G.J. Armitage and K.M. Adams, "Packet reassembly during cell loss," IEEE Network, September 1993, pp.26-34.

[2] H.J. Chao, "A novel architecture for queue management in the ATM network," IEEE J. on Select. Areas in Commun., Vol. 9, No. 7, September 1991, pp.11101118. 
[3] D. Ferrari and D. Verma, "A scheme for real-time channel establishment in widearea networks," IEEE J. on Select. Areas in Commun., Vol. 8, No. 3, April 1990, pp.368-379.

[4] J. G. Kemeny and J. L. Snell, Finite Markov Chains, Springer-Verlag, New York, 1976.

[5] H. Kröner, G. Hébuterne, P. Boyer and A. Gravey, "Priority Management in ATM switching nodes," IEEE J. on Select. Areas in Commun., Vol. 9, No. 3, April 1993, pp.418-427.

[6] H. Lee, C.G. Park, and Y.H. Kim, "Providing multiclass Quality of Service in shared ATM output multiplexer," Proceedings of IEEE GLOBECOM'96, pp.424-431, 1996.

[7] S. Ramesh, C. Rosenberg and A. Kumar, "Revenue maximization in ATM networks using the CLP capability and buffer priority management," IEEE/ACM Trans. on Networking, Vol.4, No.6, December 1996, pp.941-950.

[8] J.A. Shormans, J.M. Pitts, B.R. Clements and E.M. Scharf, "Approximation to $\mathrm{M} / \mathrm{D} / 1$ for ATM CAC, buffer dimensioning and cell loss performance," Electronic Letters, 1st February 1996, Vol.32, No.3, pp.164-165.

[9] Y. Takagi, S. Hino and T. Takahashi, "Priority assignment control of ATM line buffers with multiple QoS classes," IEEE J. on Select. Areas in Commun., Vol. 9, No. 7, September 1991, pp.1078-1092.

[10] L. Tassiulas, Y.C. Hung and S.S. Panwar, "Optimal buffer control during congestion in an ATM network node," IEEE/ACM Trans. on Networking, Vol. 2, No. 4, August 1994, pp.374-386.

\section{BIOGRAPHY}

Hoon Lee obtained a B.E. and an M.E. in electronics in 1984 and 1986, respectively, both at Kyoung-pook National University, Daegu, Korea. He obtained a Ph.D. in electrical and communications in 1996 at Tohoku University, Sendai, Japan. Since 1986, he has worked on network planning, dimensioning, and teletraffic engineering, with a particular interest in the field of ATM traffic modeling, traffic control, performance analysis, and QoS guarantee. After returning from Ph.D. program, he is also interested in the performance modeling and assessment for the wireless ATM networks. Dr. Lee is a member of IEEE and KITE of Korea. 